\title{
CODEX SCHØYEN 2650
}

\author{
James M. Leonard
}

Codex Schøyen 2650 (hereafter, mae $^{2}$ ) is a fragmentary yet substantial manuscript of Matthew's Gospel. It was written in a rare dialect of Coptic (Middle Egyptian). This thesis is the first substantial textcritical assessment of its implied underlying Greek text.

$\mathrm{Mae}^{2}$ is significant for multiple reasons. First, it is often cited as having an early Fourth Century date, and as such, eleven of its chapters in their entirety, and a large number of verses elsewhere, may be the earliest witnesses to those respective parts of Matthew's Gospel. Secondly, it provides substantial attestation of a minor Coptic dialect which was hardly known until the second half of the Twentieth Century. Thirdly, its subdialect is hitherto unattested. Fourthly, it is independent from all other Coptic versions of Matthew. Fifthly, the text of $\mathrm{mae}^{2}$ is probably one of the earliest Middle Egyptian Coptic translations, and thus from it one might infer the boundaries early translators may have had in translation technique. Finally, as I have argued, when translational phenomena are identified and accounted for, mae ${ }^{2}$ s strong alliance with both Sinaiticus and Vaticanus is evident, and this has implications for establishing the initial text of Matthew's Gospel and its early transmission history.

$\mathrm{Mae}^{2}$, s editor, H.-M. Schenke, would reject this last point about $\mathrm{mae}^{2}$ 's textual affinity with Sinaiticus and Vaticanus. Rather, Schenke argued that $\mathrm{mae}^{2}$ is significant for its attestation to an otherwise lost version of Matthew's Gospel, and not the Matthew well known through the Greek manuscript tradition ('canonical' Matthew). Indeed, in keeping with his well known assumption that the early New Testament text was transmitted chaotically, Schenke argued for a complex textual history of mae $^{2}$ involving an Aramaic Matthew that would make the two source hypothesis for the synoptic problem untenable.

1 James M. Leonard, 'Codex Schøyen 2650: Discerning a Coptic Manuscript's Witness to the Early Text of Matthew's Gospel' (Ph.D. dissertation, Cambridge University, 2012). Supervisor: P. J. Williams. 
Initially, I was in fact quite impressed by Schenke's exposition of $\mathrm{mae}^{2 \text {, }} \mathrm{s}$ strikingly different readings, such as the women going to the tomb 'in the night of the Sabbath, early in the morning at the hour of light, while the stars were still above', or that the guards at the tomb 'arose like dead men' $(28: 1,4)$, or the shift from third person to first person so that Jesus conveys, 'If $I$ had not made those days short, no flesh would be saved. But on account of $m y$ elect, $I$ will make those days short.' Moreover, Schenke's retroversion seemed to suggest that $\mathrm{mae}^{2}$ is so different from our familiar Matthew as to justify its comparison to Epiphanius' characterisation of the Gospel of the Ebionites as false and mutilated. Further into my research, however, as I compared mae 2 s Coptic text with the Greek text and the other Coptic versions, I had five realisations which led me to reject Schenke's views.

First, I found that mae $^{2}$ has too much in common with our canonical Matthew to reflect an alternative gospel. This is evident in the analysis of text unaffected or minimally affected by translation. In particular, the correspondence between $\mathrm{mae}^{2}$ and our familiar Matthew in regard to sequence of pericopes and verses, content, and the occurrence of names is all too high for $\mathrm{mae}^{2}$ to be an alternative Matthew. One of my most fruitful research endeavours was to compare variants involving larger additions or omissions since such texts are transmitted without translational interference. There are fifteen such passages extant in $\mathrm{mae}^{2}$, with $\mathrm{mae}^{2}$ supporting the text of $\mathrm{NA}^{27}$ at a higher rate of agreement than most of $\mathrm{NA}^{27}$, s consistently cited manuscripts, coming second only to Sinaiticus, and tied with Vaticanus. Together these data place the underlying Greek text of mae ${ }^{2}$ well within the common textual tradition of Matthew's Gospel.

My second realisation came when I compared $\mathrm{mae}^{2}$ with manuscripts from the broader Coptic tradition. Although $\mathrm{mae}^{2}$ is independent from them, they nonetheless share many common features. On the other hand, through my intraversional analysis, I quantified the lexical and syntactical differences between $\mathrm{mae}^{2}$ and the others, and discovered that mae $^{2}$ has fewer unique elements than the well known Coptic manuscripts $\mathrm{sa}^{9}$ and $\mathrm{bo}^{\mathrm{A}}$. The fact that $\mathrm{mae}^{2}$ has so much in common with the other Coptic versions, and the fact that these other Coptic versions have as many or more unique syntactical elements as $\mathrm{mae}^{2}$, significantly undermine Schenke's argument for a hitherto lost alternative version of Matthew. 
The third realisation was that many unexpected readings actually reflect $\mathrm{mae}^{2}$ 's translational habits or Coptic convention elsewhere. This is seen generally in the choice of conjunctions (Konjunktionsnetz, e.g.

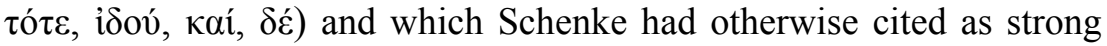
evidence to support his theory. Indeed, the lack of representation of initial $\kappa \alpha i$ and post-positive $\delta \dot{\varepsilon}$ is so pervasive as to be nearly predictable. This would commend a translational explanation over one that appeals to its underlying Greek text, especially since there seems to be no viable motive for an editor to remove them from the Greek text. Moreover, like $\mathrm{mae}^{2}$, the other Coptic versions also have Konjunktionsnetz readings that are sometimes incongruous with the Greek, giving further credence to the translational explanation. Other 'surprising' features are more or less predictable, as well. For example, mae $^{2}$ consistently avoids representing the many occurrences of -

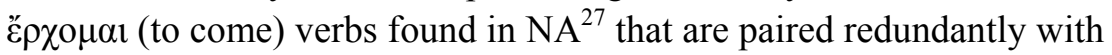
other verbs. Other features incongruous with the Greek which occur less frequently in the other Coptic versions, but more regularly in $\mathrm{mae}^{2}$, include the use of the personal independent with an inflected modifier for the formulaic 'I say to you', and the use of the periphrastic conditional for the Greek subjunctive. Examples of these and other such features abound. Other syntactical aspects are less predictable, but not unexpected in light of their treatment elsewhere in $m^{2} e^{2}$. For example, mae $^{2}$ may use the future in hypothetical contexts where the future is not used in the Greek. In some instances $\mathrm{mae}^{2}$, $\mathrm{s}$ tendencies are reflected in the simplification of complex Greek syntax, while elsewhere they are seen in the expansion of a text to clarify the Greek, or to convey information that is otherwise merely assumed by the Greek. While these phenomena occur more frequently in $\mathrm{mae}^{2}$, they nonetheless occur elsewhere in the Coptic tradition. I set forth the theoretical basis of translational expectations and the appeal to a translation theory to explain incongruities between $\mathrm{mae}^{2}$ and its underlying Greek text early in the thesis, while the interplay of theory and praxis is seen in the translational analysis of numerous blocks of text in the middle chapters of the thesis.

The fourth realisation was that, despite significant differences in syntax, mae $^{2}$ conveys the meaning of Matthew's Gospel as it is known in the Greek manuscript tradition. Interestingly, Schenke provides no examples in his introductory material which would suggest that mae ${ }^{2}$ differs significantly in its depiction of the Jesus story. As it turns out, in 
the materials I surveyed, the differences in meaning were minimal if not trivial, even though the syntactical differences were often significant.

Conclusions reached through my translational analysis of $\mathrm{mae}^{2}$ yielded a fifth realisation, one which was entirely unanticipated from the onset, but one of great significance: mae $^{2}$ often agrees with variant readings adopted by $\mathrm{NA}^{27}$ and other modern critical editions. This in itself is not surprising, given its purported date and provenance. What is surprising is that in the sixty test passages, I found that mae ${ }^{2}$ had a higher rate of agreement with $\mathrm{NA}^{27}$ than any of its allies, except for Vaticanus with which it is tied. The textual analysis also allowed a ranking of $\mathrm{mae}^{2}$, $\mathrm{s}$ closest allies, by percentage of agreement. The closest allies by far are Sinaiticus $(75 \%)$ and Vaticanus $(70 \%)$, with the next closest allies lagging by about $15 \%$. Indeed, mae ${ }^{2}$ s rate of agreement with either Sinaiticus or Vaticanus is higher than Sinaiticus and Vaticanus have with each other in the same test passages. The data and method behind these conclusions, including how the test passages were chosen, are set forth in the penultimate chapter of the thesis.

The results of the textual analysis may support the notion of an earlier text form reflected in the shared readings of $\mathrm{mae}^{2}$, Sinaiticus, and Vaticanus in Matthew's Gospel. The close affiliation of the three allows for their triangulation to help determine the better external attestation of certain variants. In some cases, mae $^{2}$ may be the only witness of the three to preserve the earlier text form, increasing the impact of $\mathrm{mae}^{2}$ s external attestation. In other cases, $\mathrm{mae}^{2}$ may join with Sinaiticus against Vaticanus, or with Vaticanus against Sinaiticus, in their claim to the purer reading. In this light, the final chapter includes a list of fourteen passages where mae ${ }^{2}$ s support for a reading against $\mathrm{NA}^{27}$ would have a significant, moderate, or marginal impact in regard to external evidence; six of the fourteen are already set in brackets in $\mathrm{NA}^{27}$. In four of these passages, $\mathrm{mae}^{2}$ may preserve the earlier textform of its allies, against Sinaiticus and Vaticanus, and accordingly, be all the more important in determining the earlier text.

The thesis is offered in the hope that a more comprehensive translational and textual analysis will be undertaken in the future. A slightly revised version is due for publication in New Testament Tools, Studies, and Documents, ed. Eldon Epp and Bart Ehrman (Leiden: Brill) in 2014. 